\title{
Surface enhanced Raman spectra of the organic nonlinear optic material: Methyl 3-(4-methoxy phenyl)prop-2-enoate
}

\author{
D SAJAN $^{\mathrm{a} 1}$ *, I HUBERT JOE ${ }^{\mathrm{a}}$, V S JAYAKUMAR ${ }^{\mathrm{a}}$ and JACEK ZALESKI ${ }^{\mathrm{b}}$ \\ ${ }^{a}$ Centre for Molecular and Biophysics Research, Department of Physics, Mar Ivanios College, \\ Thiruvananthapuram 695015 \\ ${ }^{\mathrm{b}}$ Institute of Chemistry, University of Opole, Olesksa 48 45-052 Opole, Poland \\ ${ }^{1}$ Present Address: Department of Physics, V.P.S.H.S.S. for Boys, Venganoor, \\ Thiruvananthapuram 695523 \\ e-mail: dsajand@gmail.com
}

MS received 18 May 2008; revised 17 June 2008

\begin{abstract}
The surface geometry of methyl 3-(4-methoxy phenyl)prop-2-enoate molecule was studied by analysis of the SERS spectra adsorbed on silver colloid surfaces. For a reliable analysis of the SERS spectrum, we also performed density functional theoretical calculations. The absence of a C-H stretching vibrations and the observed $\mathrm{C}-\mathrm{H}$ out-of-plane bending modes suggest that the MMP molecule may be adsorbed in a flat on orientation to the surface. The SERS spectral studies predict a tilted orientation of ethylenic bridge with respect to the phenyl ring.
\end{abstract}

Keywords. Adsorption behaviour; SERS; methyl 3-(4-methoxy phenyl)prop-2-enoate; silver nanoparticles; DFT.

\section{Introduction}

The elucidation of the adsorption mechanism of organic compounds on metal surfaces is of fundamental as well as practical interest. ${ }^{1-2}$ An increased understanding of the interaction of the molecules with metal surfaces has been accompanied by a growing awareness that many surface-adsorbate interactions have their analogues in the field of organomettalic and co-ordination chemistry. Surface-enhanced Raman scattering (SERS), using silver, gold, or other noble metals as substrates, ${ }^{3-5}$ is a powerful technique for studying the adsorption behaviour of molecules on substrates, revealing the molecules' orientation and the mechanism of interaction of the molecules with the surface of the substrate in which researchers are interested. ${ }^{6-7}$ SERS is widely used to elucidate information about the behaviour of biomolecules adsorbed at the metal surfaces, orientation of adsorbed species and the changes in the orientation induced by external factors. In addition, the adsorption of molecules on metal particles reduces the fluorescence background and hence the technique is useful in the study of biological samples. ${ }^{8-10}$ Most reported studies pertaining

\footnotetext{
*For correspondence
}

to the adsorbate molecules, possessing one or more $\sigma$-donor atoms such as $\mathrm{N}, \mathrm{O}$ and $\mathrm{S}$, many of which possess potential $\pi$-donor systems such as an aromatic ring, which could compete for interaction with the surface. The enhancement of selective vibrational modes and band shifts observed in SERS have usually been explained in terms of the charge-transfer model, and are found to be sensitive to the orientation of the molecules with respect to the surface. ${ }^{11-13}$ The technique is therefore expected to provide interesting information on the sites through which the interaction takes place and also the molecular orientation with respect to the metal surfaces. The present study reports the SERS spectral analysis of methyl 3-(4methoxy phenyl)prop-2-enoate (MMP) supported by density functional computations (DFT) to derive information about the adsorption geometry and other molecular bonding features.

\section{Experimental}

Single crystals of methyl 3-(4-methoxyphenyl)prop2-enoate (figure 1) were prepared in a methanol solution by a slow evaporation technique. ${ }^{14}$ Colourless transparent crystals of title compound were obtained 
within 2 weeks. Repeated recrystallisation yielded good quality crystals. The single crystals of maximum size of around $0.6 \mathrm{~mm}$ were obtained.

\subsection{Preparation of silver colloid}

Silver colloid was prepared by reduction of silver nitrate by sodium citrate, using the Lee and Meisel's method. ${ }^{15}$ In $500 \mathrm{ml}$ of deionized water, $90 \mathrm{mg}$ of silver nitrate was dissolved and the solution was heated to boiling. Then $10 \mathrm{ml}$ of a $1 \%$ trisodium citrate aqueous solution was added into the boiling silver nitrate solution drop-wise, accompanied by vigorous stirring. The mixed solution was kept boiling for a further $10 \mathrm{~min}$. Finally, a green-gray silver colloid was obtained, which was stable for several days or weeks. The stability of the colloid was ascertained using an Ocean Optics model S2000 fibre optic UV-visibleNIR spectrometer.

\subsection{Instrumentation}

The NIR FT-Raman spectrum (figure 2) was obtained on a RFS 100/s BRUKER spectrophotometer. $\mathrm{Nd}$ : YAG laser at $1064 \mathrm{~nm}$ with an output of $300 \mathrm{~mW}$ was used as the exciting source with liquid nitrogen cooled Ge-diode detector. 1000 scans were accumulated with a total registration time of about $30 \mathrm{~min}$. The spectral resolution after apodization was $6 \mathrm{~cm}^{-1}$. A correction according to the fourth power scattering factor was performed, but no correction to instrument was done. The upper limit for the Raman shift is $3500 \mathrm{~cm}^{-1}$ due to the detector sensitivity and the lower Raman shift is around $10 \mathrm{~cm}^{-1}$ due to the Raleigh line cut-off by a notch filter.

SERS measurements were performed on a single stage imaging spectroscope (ISA Jobin-Yvon Spex HR-320, f/4.1) fitted with a liquid nitrogen cooled CCD detector (Spectrum one, $2000 \times 800$ pixels with

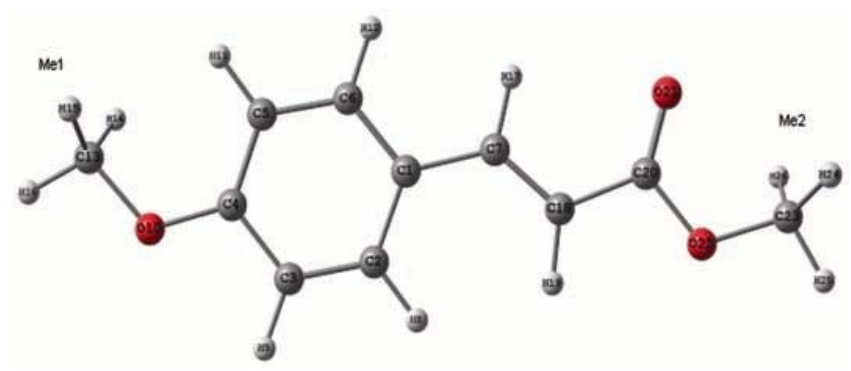

Figure 1. Molecular structure of MMP. an active area of $30 \times 12 \mathrm{~mm}$ ). The spectra were recorded with a $600 \mathrm{~g} / \mathrm{mm}$ grating blazed at $1 \mu \mathrm{m}\left(7 \mathrm{~cm}^{-1}\right)$. NIR laser excitation of $785 \mathrm{~nm}$ was provided by an SDL-8350 diode laser. To a $5 \mathrm{ml}$ of silver colloid an equal volume of $10^{-4} \mathrm{M}$ aqueous solution of MMP was added. The resultant solution having dark green colour was transferred to quartz cuvettes and the SERS spectra is recorded (figures 3 and 4).

\section{Computational details}

DFT calculations were carried out using the A.8 revision of the GAUSSIAN 98 program package. ${ }^{16}$ All calculations, which include: geometry optimizations and vibrational spectra were performed on isolated systems using the Becke's three parameter B3LYP exchange-correlation functional. ${ }^{17}$ The $6-31 \mathrm{G}(\mathrm{d})$ basis set $^{18}$ was chosen as a compromise between accuracy and applicability to large molecules. Molecular geometries were fully optimized by Berny's optimization algorithm using redundant internal coordinates. All optimized structures were confirmed to be minimum energy conformations. Harmonic vibrational wave numbers were calculated using analytic second derivatives to confirm the convergence to minima on the potential surface and to evaluate the zero-point vibrational energies. At the optimized structure of the examined species no imaginary frequency modes were obtained, proving that a true minimum on the potential energy surface was found. The optimum geometry was determined by minimizing the energy with respect to all geometrical parameters without

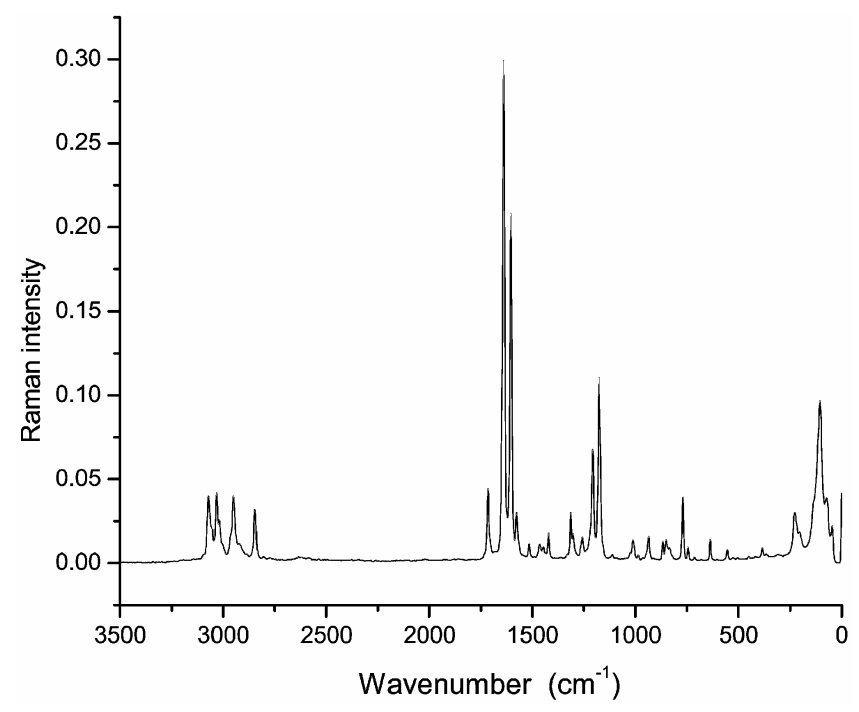

Figure 2. NIR-FT Raman spectrum of MMP. 


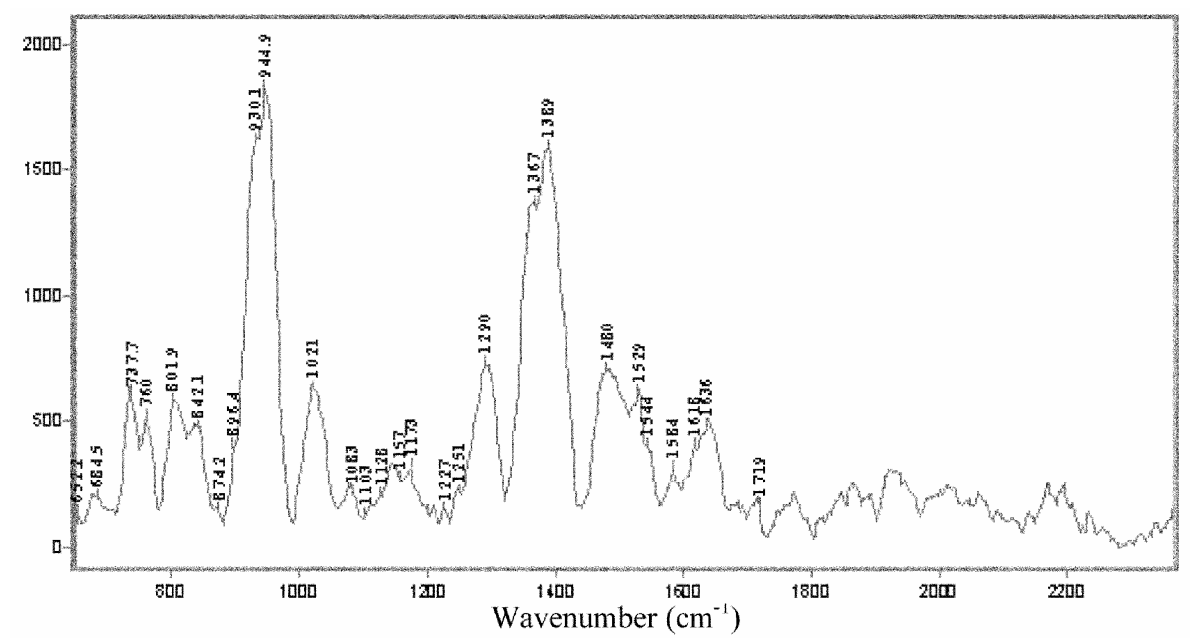

Figure 3. NIR-FT SERS spectrum of MMP $\left(2400-600 \mathrm{~cm}^{-1}\right)$.

imposing molecular symmetry constraints. The calculated harmonic vibrational wavenumbers were scaled down uniformly by a factor of 0.96 as recommended by Scott and Radom. ${ }^{19}$

\section{Results and discussions}

The SERS spectrum of MMP has been recorded and the vibrational analysis is performed by comparing it with the normal Raman spectrum. The vibrational spectrum calculated at B3LYP/6-311G $(d, p)$ is used to determine the vibrational modes unambiguously. The calculated vibrational wavenumbers, measured SERS and Raman band positions and their tentative assignments are given in table 1. The adsorption mechanism of an adsorbate can be deduced from its SERS spectrum. The orientation of the adsorbate on the metal surface will depend on the active sites through which the interaction takes place. The chemically possible orientations of the MMP molecular ion with respect to the silver surface can be envisaged: 'flat' or lying down on the metal surface through bonding with the ring system or 'standing up' (end-on) with bonding through the lone pair of the oxygen atom with silver. ${ }^{20-23}$ The orientation of the molecule on the silver surface can be inferred from aromatic $\mathrm{C}-\mathrm{H}$ stretching vibrations, ring stretching vibrations, the ring breathing mode, in plane and out-of-plane vibrations and the SERS surface selection rule.

The ring stretching vibrational modes of the phenyl ring with vibration numbers $14,19 \mathrm{a}, 19 \mathrm{~b}, 8 \mathrm{a}$ and $8 \mathrm{~b}$ among which the degenerate vibrations are observed around $1600 \mathrm{~cm}^{-1} \cdot{ }^{23-25}$ The vibrational mode corre- sponding to the band at a wavenumber of $1618 \mathrm{~cm}^{-1}$ and $1585 \mathrm{~cm}^{-1}$ in SERS is also observed in normal Raman spectrum at $1604 \mathrm{~cm}^{-1}$ and $1577 \mathrm{~cm}^{-1}$ as a strong band. Here the ring mode is blue shifted by around $10 \mathrm{~cm}^{-1}$ along with the substantial band broadening in SER spectrum. This indicates the direct interaction of benzene ring moiety with the metal surface, as well documented in literature ${ }^{5-7}$ and the probability of direct ring $\pi$ orbital to metal interaction should be high. ${ }^{23-25}$ This can be interpreted as the bond weakening in the benzene ring system caused by the back donation of the metal d electrons to the benzene ring antibonding $\pi^{*}$ orbitals. ${ }^{25}$ The modes corresponding to $19 \mathrm{a}$ can be found at $1516 \mathrm{~cm}^{-1}$ in Raman spectrum are down-shifted in SERS spectrum at $1529 \mathrm{~cm}^{-1}$ and their bandwidths are hardly affected. The SERS band at $1389 \mathrm{~cm}^{-1}$ is up-shifted by about $30 \mathrm{~cm}^{-1}$ and the band width is decreased with respect to the corresponding band at $1419 \mathrm{~cm}^{-1}$ in Raman spectrum. From the observed both down-shifted and up-shifted peaks, it is difficult to make an unambiguous orientation of the absorbed molecules using ring stretching vibrations.

The $\mathrm{C}=\mathrm{C}$ stretching, observed as a strong characteristic band in cinnamic acid derivatives, can be seen in MMP at $1639 \mathrm{~cm}^{-1}$ in Raman spectrum as a strong band. This band is blue-shifted to $1647 \mathrm{~cm}^{-1}$ in SERS spectrum followed by an intensity reduction, which predicts a tilted orientation of the ethylenic bridge with respect to the phenyl ring. The ethylenic bridge is slightly out of the plane with the $\mathrm{O}-\mathrm{Me}$ bond of $\mathrm{CH}_{3}$ on the metal surface, favoured by $\pi$ orbital metal surface interaction. ${ }^{23-25}$ The $\mathrm{CH}_{3}$ asym- 


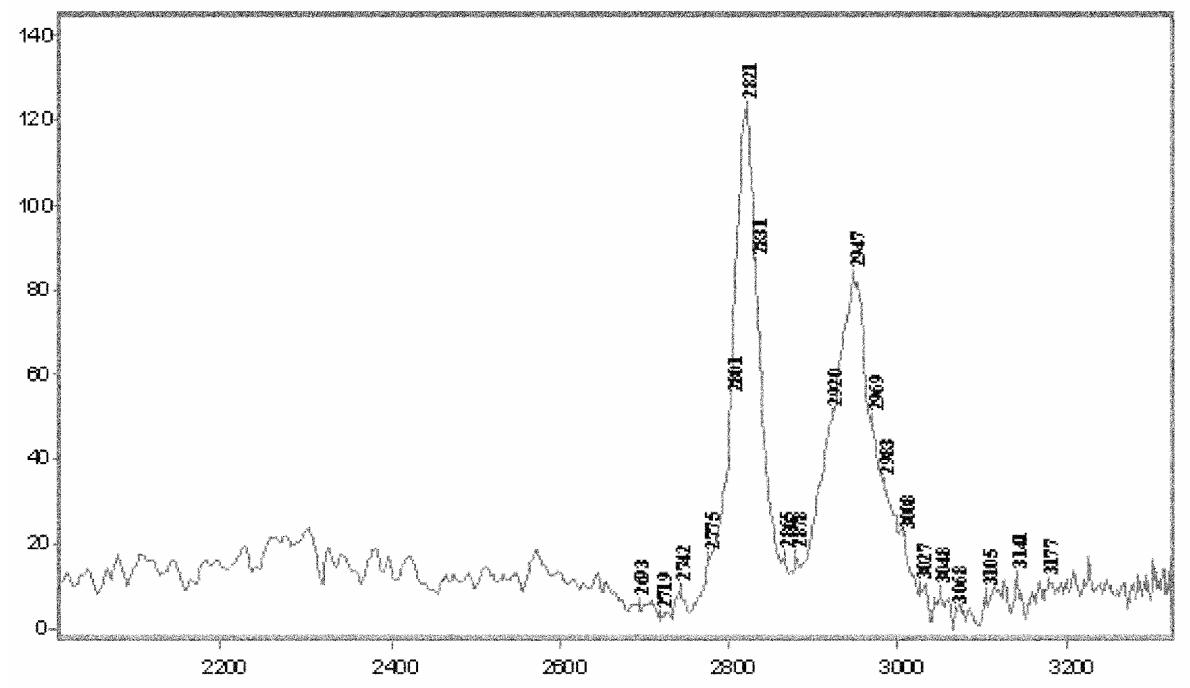

Figure 4. NIR-FT SERS spectrum of MMP (2200-3200 $\left.\mathrm{cm}^{-1}\right)$.

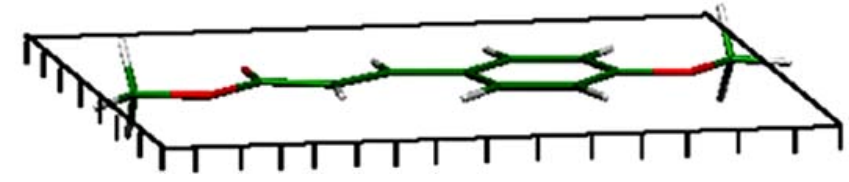

Figure 5. Schematic model for the adsorption geometry of MPP on a colloidal sliver surface.

metric bending mode can be observed as a medium intense in MMP at $1480 \mathrm{~cm}^{-1}$ in SERS, which confirms a flat orientation of the $\mathrm{CH}_{3}$ in the adsorbed molecule with respect to the metal surface. Moreover, the medium intense band at $1716 \mathrm{~cm}^{-1}$ in the NIR-FT Raman spectrum assigned to the carbonyl stretching vibration is not observed in the SERS spectrum and this also supports the above assumption. The carbonyl stretching appears only as a very weak band at $1719 \mathrm{~cm}^{-1}$ in the SERS spectrum. The weakening of this carbonyl stretching vibration indicates that the $\mathrm{C}=\mathrm{O}$ is not in direct interaction with the silver surface.

In the case of aromatic molecules, the intensity of out-of-plane vibrational modes increases substantially relative to the in-plane vibrational modes when the absorbate orientation is altered from vertical to flat on orientation. ${ }^{20-25}$ The in-plane vibrational modes occur both in Raman spectrum and SERS spectra in the region $1300-1000 \mathrm{~cm}^{-1}$. Some of the in-plane vibrational modes are not observed in the SERS spectrum. The intensity of the SERS band is less than that of the band in Raman spectrum. Similarly, aromatic out-of-plane $\mathrm{C}-\mathrm{H}$ bands with respect to the inplane bending mode increases as the molecule is adsorbed on almost flat to the silver surface. ${ }^{23-25}$ The absence of some of the in-plane bending modes also indicates that the probability of the absorption of the MMP molecule in a flat on orientation is greater.

The SERS spectrum of the molecules, which are flat on the metal surface, would contain strong out-ofplane vibrations. For a benzene molecule lying flat on the surface, the intensity of the $\mathrm{C}-\mathrm{H}$ stretching mode would be almost zero, since the $\mathrm{C}-\mathrm{H}$ stretching vibrations do not have contributions from $\alpha_{\mathrm{zz}}$, $\alpha_{\mathrm{xz}}$ and $\alpha_{\mathrm{yz}}$ Raman polarizability components which would result in strong SERS intensity. ${ }^{24}$ However, the out-of-plane vibrations do have large contributions from these Raman polarizability components. In MMP shows four $\mathrm{C}-\mathrm{H}$ out-of-plane bending vibrations at $930 \mathrm{~cm}^{-1}, 896 \mathrm{~cm}^{-1}, 842 \mathrm{~cm}^{-1}$ and $760 \mathrm{~cm}^{-1}$ in SERS spectrum having medium intensity. However, it does not show any $\mathrm{C}-\mathrm{H}$ stretching vibrations. The entire $\mathrm{C}-\mathrm{H}$ out-of-plane bending modes belong to an ' $A$ ' species, which has the polarizability components $\alpha_{\mathrm{xz}}$ and $\alpha_{\mathrm{yz}}$. The observed C-H out-of-plane bending modes, obtain their SERS intensity from these Raman polarizability components. The absence of a $\mathrm{C}-\mathrm{H}$ stretching vibrations and the observed $\mathrm{C}-$ $\mathrm{H}$ out-of-plane bending modes suggest that the MMP molecule may be adsorbed in a flat on orientation to the surface (figure 5). The enhancement methyl asymmetric $\left(2947 \mathrm{~cm}^{-1}\right)$, symmetric stretch- 
Table 1. Calculated vibrational wavenumbers, measured SERS and Raman band positions $\left(\mathrm{cm}^{-1}\right)$ and assignments for methyl 3(4-methoxy phenyl) prop2-enoate.

\begin{tabular}{|c|c|c|c|}
\hline B3LYP/6311G(d, p) $V_{\text {cal }}$ & $v_{\mathrm{SERS}}$ & $\nu_{\text {Raman }}$ & Assignments \\
\hline 2971 & $2960 \mathrm{sh}$ & $2947 \mathrm{~m}$ & $\mathrm{CH}_{3}$ asym. stretch \\
\hline 2910 & $2846 \mathrm{~m}$ & $2821 \mathrm{~s}$ & $\mathrm{CH}_{3}$ sym. stretch \\
\hline 1727 & $1716 \mathrm{~m}$ & $1719 w$ & $\mathrm{C}=\mathrm{O}$ stretch \\
\hline 1632 & 1639 vvs & $1636 \mathrm{~m}$ & $\mathrm{C}_{7}=\mathrm{C}_{8}$ stretch \\
\hline 1602 & $1604 \mathrm{vs}$ & $1618 \mathrm{sh}$ & 8 a ring stretch \\
\hline 1558 & $1577 \mathrm{w}$ & $1584 \mathrm{w}$ & $8 \mathrm{~b}$ ring stretch \\
\hline 1504 & $1513 \mathrm{vw}$ & $1529 \mathrm{~m}$ & 19a ring stretch \\
\hline 1462 & & $1480 \mathrm{~m}$ & $\mathrm{CH}_{3}$ asym. bend \\
\hline 1415 & $1419 \mathrm{vw}$ & $1389 \mathrm{~s}$ & $19 \mathrm{~b}$ ring stretch \\
\hline 1340 & & $1367 \mathrm{~s}$ & $\mathrm{CH}_{3}$ sym. bend \\
\hline 1303 & 1304 vvw & $1290 \mathrm{~m}$ & $3, \mathrm{C}-\mathrm{H}$ i.p bend \\
\hline 1171 & $1176 \mathrm{~m}$ & $1173 \mathrm{w}$ & $\mathrm{CH}_{3}$ rock \\
\hline 1155 & & $1157 \mathrm{w}$ & $9 \mathrm{~b}, \mathrm{C}-\mathrm{H}$ i.p bend \\
\hline 1099 & & $1083 \mathrm{w}$ & $18 \mathrm{~b}, \mathrm{C}-\mathrm{H}$ i.p bend \\
\hline 1016 & $1024 \mathrm{w}$ & $1021 \mathrm{~m}$ & $\mathrm{C}_{18}-\mathrm{H}_{19}$ i.p bend \\
\hline 931 & & $945 \mathrm{vs}$ & $\mathrm{C}-\mathrm{O}=\mathrm{O}$ i.p bend \\
\hline 926 & $935 \mathrm{w}$ & $930 \mathrm{~s}$ & $\mathrm{C}_{18}-\mathrm{H}_{19}$ o.p bend \\
\hline 915 & & $896 \mathrm{sh}$ & $17 \mathrm{~b}, \mathrm{C}-\mathrm{H}$ o.p bend \\
\hline 831 & $840 \mathrm{vVw}$ & $842 \mathrm{~m}$ & $10 \mathrm{a}, \mathrm{C}-\mathrm{H}$ o.p bend \\
\hline 792 & & $801 \mathrm{~m}$ & 1 ring breadth \\
\hline 755 & $769 \mathrm{~m}$ & $760 \mathrm{~m}$ & $\mathrm{C}-\mathrm{H}$ o.p bend \\
\hline 732 & $744 \mathrm{vVw}$ & $738 w$ & $\mathrm{C}-\mathrm{H}$ o.p bend \\
\hline 678 & & $683 \mathrm{w}$ & $\mathrm{C}_{18}-\mathrm{H}_{19}$ i.p bend \\
\hline 2971 & $2960 \mathrm{sh}$ & $2947 \mathrm{~m}$ & $\mathrm{CH}_{3}$ asym. stretch \\
\hline 2910 & $2846 \mathrm{~m}$ & $2821 \mathrm{~s}$ & $\mathrm{CH}_{3}$ sym. stretch \\
\hline 1727 & $1716 \mathrm{~m}$ & $1719 \mathrm{w}$ & $\mathrm{C}=\mathrm{O}$ stretch \\
\hline 1632 & 1639 vvs & $1636 \mathrm{~m}$ & $\mathrm{C}_{7}=\mathrm{C}_{8}$ stretch \\
\hline 1602 & $1604 \mathrm{vs}$ & $1618 \mathrm{sh}$ & $8 \mathrm{a}$ ring stretch \\
\hline 1558 & $1577 \mathrm{w}$ & $1584 \mathrm{w}$ & $8 \mathrm{~b}$ ring stretch \\
\hline 1504 & $1513 \mathrm{vw}$ & $1529 \mathrm{~m}$ & 19a ring stretch \\
\hline 1462 & & $1480 \mathrm{~m}$ & $\mathrm{CH}_{3}$ asym. bend \\
\hline 1415 & $1419 \mathrm{vw}$ & $1389 \mathrm{~s}$ & $19 \mathrm{~b}$ ring stretch \\
\hline 1340 & & $1367 \mathrm{~s}$ & $\mathrm{CH}_{3}$ sym. bend \\
\hline 1303 & 1304 vvw & $1290 \mathrm{~m}$ & $3, \mathrm{C}-\mathrm{H}$ i.p bend \\
\hline 1171 & $1176 \mathrm{~m}$ & $1173 \mathrm{w}$ & $\mathrm{CH}_{3}$ rock \\
\hline 1155 & & $1157 \mathrm{w}$ & $9 b, \mathrm{C}-\mathrm{H}$ i.p bend \\
\hline 1099 & & $1083 \mathrm{w}$ & $18 \mathrm{~b}, \mathrm{C}-\mathrm{H}$ i.p bend \\
\hline 1016 & $1024 \mathrm{w}$ & $1021 \mathrm{~m}$ & $\mathrm{C}_{18}-\mathrm{H}_{19}$ i.p bend \\
\hline 931 & & $945 \mathrm{vs}$ & $\mathrm{C}-\mathrm{O}=\mathrm{O}$ i.p bend \\
\hline 926 & $935 \mathrm{w}$ & $930 \mathrm{~s}$ & $\mathrm{C}_{18}-\mathrm{H}_{19}$ o.p bend \\
\hline 915 & & $896 \mathrm{sh}$ & $17 \mathrm{~b}, \mathrm{C}-\mathrm{H}$ o.p bend \\
\hline 831 & $840 \mathrm{vVw}$ & $842 \mathrm{~m}$ & $10 \mathrm{a}, \mathrm{C}-\mathrm{H}$ o.p bend \\
\hline 792 & & $801 \mathrm{~m}$ & 1 ring breadth \\
\hline 755 & $769 \mathrm{~m}$ & $760 \mathrm{~m}$ & $\mathrm{C}-\mathrm{H}$ o.p bend \\
\hline 732 & 744 vvw & $738 \mathrm{w}$ & $\mathrm{C}-\mathrm{H}$ o.p bend \\
\hline 678 & & $683 \mathrm{w}$ & $\mathrm{C}_{18}-\mathrm{H}_{19}$ i.p bend \\
\hline
\end{tabular}

ing $\left(2821 \mathrm{~cm}^{-1}\right)$, asymmetric bending $\left(1480 \mathrm{~cm}^{-1}\right) \mathrm{um}$ brella $\left(1367 \mathrm{~cm}^{-1}\right)$ and rocking mode $\left(1173 \mathrm{~cm}^{-1}\right)$ further support the orientation of MMP on a silver surface is flat on orientation of the benzene ring moiety on a silver substrate.

\section{Conclusion}

The comparative study of normal Raman spectrum with the SERS along with the scaled DFT calculations predicts the adsorption geometry of MMP on 
silver surface. The SERS spectral analysis indicated that the MMP molecules should be bound to silver as flat on orientation of the benzene ring moiety on a silver substrate. The absence of a $\mathrm{C}-\mathrm{H}$ stretching vibrations and the observed $\mathrm{C}-\mathrm{H}$ out-of-plane bending modes suggest that the MMP molecule may be adsorbed in a flat on orientation to the surface. The SERS spectral studies predict a tilted orientation of ethylenic bridge with respect to the phenyl ring.

\section{References}

1. Cîntã S, Vogel E, Maniu D, Aluas M, Iliescu T, Cozar O and Kiefer W 1999 J. Mol. Struct. 482-483 679

2. Procházka M, Hanzliková J, Štepánek J and Baumruk V 1997 J. Mol. Struct. 410-411 7

3. Greaves S J and Griffith W P 1991 Spectrochim. Acta Part A47 133

4. Zhang P X, Gao X P and Zhuang W P 1985 Acta Phys. Sin. 341603

5. Sánchez-Cortés S and García-Ramos J V $2000 \mathrm{~J}$. Colloid Interface Sci. 23198

6. Sánchez-Cortés S, Francioso O, Ciavatta C, GarcíaRamos J V and Gessa C 1998 J. Colloid Interface Sci. 198308

7. Kneipp K, Casari R R and Wang Y 1994 Appl. Spectrosc. 48951

8. Wu Y Q, Zhao B, Xu W Q, Li G W and Li B 1999 Langmuir 151247
9. Muniz-Miranda M 2002 Vibrat. Spectrosc. 29229

10. Muniz-Miranda M, Neto N and Sbrana G $1997 \mathrm{~J}$. Mol. Struct. 410-411 205

11. Hesse E and Creighton J A 1999 Chem. Phys. Lett. 303101

12. Sallack J, Maiti A K, Aroca R and Menendez J R 1997 J. Mol. Struct. 410-411 217

13. Chen Y H and Yeh C Y 2002 Colloids Surf. A197 133

14. Maciej Bujak, Jacek Zaleski, Victor Prezhdo, BorisUspienskij 2002 Acta Crystallogr. C58 O76

15. Lee P C and Meisel D 1982 J. Phys. Chem. 86 3391

16. Gaussian 98, revision A.9. Frisch M J et al Gaussian, Inc. Pittsburgh PA, 1998

17. Foresman J B and Frisch A 1996 Exploring chemistry with electronic structure methods; second edition, Gaussian Inc: Pittsburgh, USA

18. Becke A D 1999 J. Chem. Phys. 985648

19. Anthony P Scott and Leo Radom 1996 J. Phys. Chem. 10016503

20. Moskovits M 1982 J. Chem. Phys. 774408

21. Moskovits M 1988 J. Phys. Chem. 926327

22. Creighton J A 1983 Surf. Sci. 124209

23. Giese B and McNaughton D 2002 J. Phys. Chem. B106 1461

24. Sang Woo Joo, Sang Woo Han, Hyouk Soo Han and Kwan Kim 2000 J. Raman Spect. 31145

25. Dong Hee Son, Sang Jung Ahn, Young Joo Lee and Kwan Kim 1994 J. Phys. Chem. 988488

26. Meic Z, Suste T, Baranovic G, Smrecki V, Holly S and Keresztury G 1995 J. Mol. Struct. 348229 